\title{
Werkstätten in der Kritik
}

\section{Beschäftigungsangebot der Zukunft für Menschen mit Behinderungen}

\author{
Alexander Vater und Laurenz Aselmeier
}

Die Werkstätten für behinderte Menschen gelten in Deutschland als wichtige Einrichtung zur Einlösung des gesetzlichen Auftrags, auch Menschen mit einer Behinderung die Teilhabe am Arbeitsleben zu sichern. Die Stellung der Werkstätten scheint jedoch angesichts neuer gesetzlicher Bestimmungen, wie dem Persönlichen Budget, und steigendem Bedarf an Werkstattplätzen infrage gestellt.

Sind Werkstätten für behinderte Menschen noch das Beschäftigungsangebot der Zukunft? Diese Frage wurde über lange Jahre nahezu uneingeschränkt mit Ja beantwortet. Es war unbestritten, dass Teilhabe am Arbeitsleben für Menschen mit Behinderung oder psychischer Erkrankung dort verwirklicht werden konnte.

Seit gut einem Jahr werden Werkstätten für behinderte Menschen zunehmend hinterfragt. Ursache für die aufkommende Kritik sind die zunehmende Anzahl von Beschäftigten und die damit verbundenen steigenden Kosten (vgl. BAGüS/con_sens 2007). Bundesweit gibt es derzeit circa 250.000 Menschen mit Behinderungen, die in Werkstätten eine berufliche Bildung und Teilhabe am Arbeitsleben erfahren. Legt man die Steigerungsraten der letzten Jahre zugrunde, so ist vorauszusehen, dass im Jahr 2010 fast 300.000 Werkstättenplätze benötigt werden (vgl. Vater 2008).

Dr. Alexander Vater ist stellvertretender Geschäftsführer der Johannes Anstalten Mosbach und Vorstandsmitglied des

Bundesverbands evangelische

Behindertenhilfe e. V.

Internet http://http://www.jamos.de

Dr. Laurenz Aselmeier ist Referent des

Bundesverbands evangelische

Behindertenhilfe e. V.

Internet http://www.beb-ev.de
Der zunehmende Bedarf an Werkstattplätzen lässt sich im Wesentlichen auf drei Entwicklungen zurückführen: Die Zunahme von behinderten oder von Behinderung bedrohten Kindern und Jugendlichen, die fehlenden Eingliederungsmöglichkeiten auf dem regulären Arbeitsmarkt und die Zunahme des Anteils der Leistungsberechtigten mit psychischen Erkrankungen in den Werkstätten für behinderte Menschen (vgl. Vater 2007). Der Zuwachs von Beschäftigten hängt auch damit zusammen, dass Zugängen aus den Sonderschulen kaum Abgänge durch Verrentung gegenüberstehen. Warum die atypische Alterspyramide bei Menschen mit Behinderung oder psychischer Erkrankung in Deutschland entstanden ist, ist mit einem Blick in die deutsche Geschichte hinlänglich zu erklären.

Die traditionellen Angebote der Behindertenhilfe befinden sich seit fast 15 Jahren im Wandel. Unter den Leitmotiven der Dezentralisierung und Ambulantisierung haben viele Einrichtungen der Behindertenhilfe ihr Wohnangebot neu ausgerichtet. Die Beschäftigungsangebote wurden von diesen Veränderungsprozessen dagegen bislang kaum erfasst.

Seitens der Werkstattkritiker ist nun von Fehlsteuerung die Rede, die hauptsächlich damit begründet wird, dass es einen Automatismus des Übergangs von der Schule in die Werkstatt gibt. Außerdem wird behauptet, dass etwa fünf Prozent der Werkstattbeschäftigten »fehlplaziert « seien (vgl. BAGüS/BIH 2007). Damit wird unterstellt, dass für diese Menschen die unterstützenden Arbeitsund Beschäftigungsangebote in einer Werkstatt nicht erforderlich seien, sondern ihnen auch der freie Arbeitsmarkt offen stünde.

Wenig wird in der Kritik indes berükksichtigt, ob der sogenannte erste Arbeitsmarkt überhaupt hinreichende Beschäftigungsmöglichkeiten für Menschen mit Behinderungen vorhält. Ebenso wenig im öffentlichen Blick ist die Frage, welche Möglichkeiten zur Teilhabe am Arbeitsleben sich denn für Menschen mit schwersten Behinderungen und hohem Unterstützungsbedarf bieten.

Zwar sind in den vergangenen Jahren zahlreiche Programme mit dem Ziel aufgelegt worden, schwerbehinderte Menschen auf den ersten Arbeitsmarkt zu integrieren. Allerdings wird bei diesen Programmen von der Vorstellung ausgegangen, dass durch zeitlich befristete Lohnkostenzuschüsse eine dauerhafte Beschäftigung auf dem allgemeinen Arbeitsmarkt erreicht werden kann. Diese Hoffnung hat sich in den meisten Fällen nicht erfüllt. Ohne dauerhafte Unterstützungsleistungen, beispielsweise in Form eines Minderleistungsausgleichs, werden die Vermittlungsquoten nicht deutlich anzuheben sein. Integrationsprojekte könnten eine geeignete Alternative sein, jedoch sind diese Projekte von der Einnahmesituation abhängig und somit nicht dauerhaft finanziert. Die aus Sicht behinderter Menschen notwendige dauerhafte Finanzierungsabsicherung fehlt ebenso wie ein individueller Rechtsanspruch auf diese Leistungen.

Deshalb wird die Werkstatt auch in Zukunft für den Großteil geistig und körperbehinderter, aber auch seelisch beeinträchtigter Menschen die Einrichtung zur Teilhabe am Arbeitsleben sein. Werkstätten sind daher ein Beschäftigungsangebot für behinderte Menschen, das nicht wegzudenken ist. Allerdings ist unbestritten, dass die Werkstätten vor der Herausforderung stehen, ihr Angebot stärker auszudifferenzieren und in Richtung einer personenzentrierten Teilhabeförderung weiterzuentwickeln.

Dazu gehört auch, dass Werkstätten ihre Anstrengungen verstärken, Menschen mit Behinderungen dabei zu unterstützen, Zugang zum allgemeinen Arbeitsmarkt $\mathrm{zu}$ finden. Konzepte wie Außenarbeitsgruppen und Außeneinzelarbeitsplätze, Praktika oder das Modell der virtuellen Werkstatt sollten weiter ausgebaut werden. 
Neben der Weiterentwicklung der Werkstätten müssen sich die Entwicklungsanstrengungen auf dauerhafte sozialversicherungspflichtige Beschäftigungsverhältnisse auf dem allgemeinen Arbeitsmarkt konzentrieren. Daran sind allerdings folgende Bedingungen geknüpft:
In der Konsequenz lässt sich feststellen, dass in der Kombination von individuellen Nachteilsausgleichen mit der Gewährung eines dauerhaften Minderleistungsausgleichs an Arbeitgeber und mit der Finanzierung von Arbeitsassistenz für die Beschäftigten der Schlüssel für eine Lösung liegen könnte. Die Zeichen der Zeit

\section{»Die Beschäftigungsangebote für Menschen mit einer Behinderung wurden bislang kaum vom Wandel in der Behindertenhilfe erfasst «}

- Kein Verlust des behinderungsbedingten Nachteilsausgleichs: Als behinderungsbedingte Nachteilsausgleiche gibt es besondere sozialversicherungsrechtliche Regelungen (z. B. Rentenbezug nach 20 Jahren), die jedoch an die Beschäftigung in einer anerkannten Werkstatt für Behinderte geknüpft sind. Die Übergänge auf den allgemeinen Arbeitsmarkt könnten erleichtert werden, wenn diese Regelungen auch Menschen mit Behinderungen auf dem allgemeinen Arbeitsmarkt zur Verfügung stehen würden.

- Dauerhafter Minderleistungsausgleich: Wenn man zur Kenntnis nimmt, dass behinderte Menschen auf Dauer nicht die vollen Leistungen erbringen werden, muss über einen Ausgleich etwaiger Minderleistungen nachgedacht werden. Die Chancen einer Vermittlung auf den allgemeinen Arbeitsmarkt ließen sich beträchtlich verbessern, wenn Unternehmen, die Menschen mit Behinderungen oder psychischer Beeinträchtigung einstellen, ein dauerhafter Minderleistungsausgleich gewährt würde.

- Arbeitsassistenz: Viele Menschen mit Behinderung oder psychischer Erkrankung, die eine Beschäftigung auf dem allgemeinen Arbeitsmarkt gefunden haben, benötigen für die gesamte Dauer ihres Arbeitslebens Unterstützung, um die Anforderungen des allgemeinen Arbeitsmarktes bewältigen zu können. Es sind gesetzliche Regelungen $\mathrm{zu}$ schaffen, die die Arbeitsassistenz für behinderte Menschen dauerhaft absichern. deuten darauf hin, dass gemeinsame Anstrengungen auf nationaler und internationaler Ebene unternommen werden müssen, um die verbrieften Rechte von Menschen mit Behinderung oder psychischer Erkrankung auch im Hinblick auf die Teilhabe am Arbeitsleben zu realisieren (siehe hierzu insbesondere Artikel 27 der UN-Konventionen über die Rechte behinderter Menschen).

\section{Literatur}

BAGüS/BIH (2007): Schnittstelle allgemeiner Arbeitsmarkt. Werkstatt für behinderte Menschen. Schwachstellen und Lösungsperspektiven. Münster, Karlsruhe,

BAGüS/con_sens (2007): Kennzahlenvergleich der überörtlichen Träger der Sozialhilfe. Bericht 2005/2006. Münster, Hamburg.

ISB (2007): Entwicklung der Zugangszahlen zu WfbM. Bestanderhebung: Zwischenstand 04.12.2007. Berlin.

Vater, Alexander (2007): Gibt es zur Werkstatt für behinderte Menschen eine Alternative? In: Rechtsdienst der Lebenshilfe 01/2007.

Vater, Alexander (2008): Sind Werkstätten noch das Beschäftigungsangebot der Zukunft? Vortrag auf der BeBFachtagung »Bunte Vielfalt unter einem Dach« am 9. Juni 2008.

Vereinte Nationen (2006): Übereinkommen über die Rechte von Menschen mit Behinderungen. Quelle: www.bmas.de,

\section{Die Verfahrens-} regeln in der Anwendung

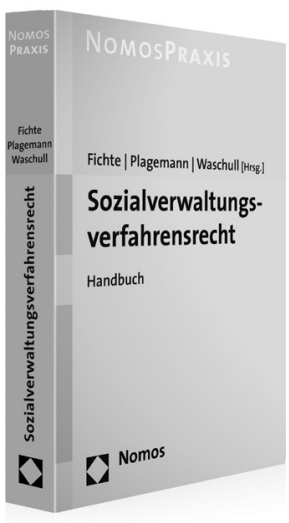

\section{Sozialverwaltungs- verfahrensrecht}

Handbuch

Herausgegeben von RiBSG Dr. Wolfgang Fichte, RA Prof. Dr. Hermann Plagemann, FAMedR u FASozR, und RiLSG Prof. Dr. Dirk Waschull 2008, 433 S., brosch., 49,- $€$ ISBN 978-3-8329-2610-6

Das Handbuch berücksichtigt die verschiedenen Herangehensweisen der Sozialbehörden, Sozialgerichte und der Anwaltschaft. Anhand typischer Anwendungsprobleme stellen die Autoren die Verbindung von Verfahrensrecht und materiellem Recht her. Die Unterschiede zwischen dem Sozialverwaltungsverfahren nach SGB $X$ und dem VwVfG-Verfahren werden verdeutlicht.

Zahlreiche Beispiele, Antragsmuster, Gebührenhinweise sowie Ausführungen zum (einstweiligen) Rechtsschutz erhöhen den praktischen Nutzen.

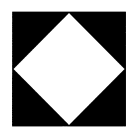

Nomos

Bitte bestellen Sie im Buchhandel oder versandkostenfrei unter $\downarrow$ www.nomos-shop.de 